\title{
TNFR2 maintains adequate IL- I 2 production by dendritic cells in inflammatory responses by regulating endogenous TNF levels
}

Innate Immunity

2014, Vol. 20(7) 712-720

(C) The Author(s) 2013

Reprints and permissions: sagepub.co.uk/journalsPermissions.nav DOI: $10.1177 / 1753425913506949$ ini.sagepub.com

SAGE

\author{
Elisabeth M Martin',*, Annika Remke',*, Eva Pfeifer', \\ Johannes Polz', Anne Pietryga-Krieger', \\ Dorothea Steffens-Weber', Marina A Freudenberg ${ }^{2}$, \\ Sven Mostböck ${ }^{1, \dagger}$ and Daniela N Männel ${ }^{1, \dagger}$
}

\begin{abstract}
Sepsis-induced immune reactions are reduced in TNF receptor 2 (TNFR2)-deficient mice as previously shown. In order to elucidate the underlying mechanisms, the functional integrity of myeloid cells of TNFR2-deficient mice was analyzed and compared to wild type (WT) mice. The capacity of dendritic cells to produce IL- I 2 was strongly impaired in TNFdeficient mice, mirroring impaired production of IL- I 2 by WT dendritic cells in sepsis or after LPS or TNF pre-treatment. In addition, TNFR2-deficient mice were refractory to LPS pre-treatment and also to hyper-sensitization by inactivated Propionibacterium acnes, indicating habituation to inflammatory stimuli by the immune response when TNFR2 is lacking. Constitutive expression of TNF mRNA in kidney, liver, spleen, colon and lung tissue, and the presence of soluble TNFR2 in urine of healthy WT mice supported the conclusion that TNF is continuously present in naïve mice and controlled by soluble TNFR2. In TNFR2-deficient mice endogenous TNF levels cannot be balanced and the continuous exposure to enhanced TNF levels impairs dendritic cell function. In conclusion, TNF pre-exposure suppresses secondary inflammatory reactions of myeloid cells; therefore, continuous control of endogenous TNF by soluble TNFR2 seems to be essential for the maintenance of adequate sensitivity to inflammatory stimuli.
\end{abstract}

\section{Keywords}

Tolerance, tumor necrosis factor, inflammation, immune suppression

Date received: 18 April 20I3; revised: 19 July 20I3; 8 August 20I3; accepted: 6 September 2013

\section{Introduction}

TNF, a key cytokine induced by inflammatory stimuli, has a wide range of biological functions. This is probably owing to the fact that it exists in two biologically active forms, as a soluble and as a trans-membrane molecule. In addition, the two forms interact differentially with two types of receptors, TNF receptor type 1 (TNFR1) and TNF receptor type 2 (TNFR2), which activate different signaling pathways. ${ }^{1}$ Both receptors can be detected on the surface of a number of different cell types, with TNFR1 being constitutively expressed on many cell types, while the expression of TNFR2 is highly regulated and mainly restricted to immune cells. $^{2}$ In addition, the two TNFRs can be cleaved off membranes and act as soluble TNF scavengers. TNFR2, in particular, is released in large quantities during inflammation and acts as functional inhibitor of TNF.

TNFR1 is the principal receptor on most cell types, triggering inflammatory signals classically attributed to

\footnotetext{
'Institute of Immunology, University of Regensburg, Regensburg, Germany

${ }^{2}$ Max-Planck-Institute for Immunobiology, Freiburg, Germany

*Joint first authors: EM Martin and A Remke contributed equally to the work.

†'oint senior authors: S Mostböck and DN Männel contributed equally to the work.
}

\section{Corresponding author:}

Daniela Männel, Institute of Immunology, University of Regensburg,

F.-J.-Strauss-Allee II, D - 93042 Regensburg, Germany.

Email: daniela.maennel@klinik.uni-regensburg.de 
TNF, such as triggering cytotoxic and inflammatory processes. $^{3,4}$ Important TNFR1-dependent signaling pathways include pathways that result in activation of NF-kB, Jun N-terminal kinase, MAPK and others. Consequently, TNFR1-deficient mice are resistant to endotoxic shock and demonstrate an impaired defense to bacterial pathogens. ${ }^{5,6}$

Soluble TNFR2 is generally considered to attenuate TNF responses by scavenging TNF. ${ }^{4}$ The physiological role of signal transmission through TNFR2, however, is not entirely clear and genetic ablation of the gene for TNFR2 did not result in a mouse line with a strong phenotype. Membrane TNFR2 was found to enhance TNFR1-induced apoptosis, first believed to be due to a phenomenon called 'ligand passing ${ }^{7}$ and, later, to intracellular signaling. ${ }^{8,9}$ With respect to inflammatory pathways, both synergistic and antagonizing activities of TNFR1 and TNFR2 have been described. The differential interaction of membrane-bound versus soluble TNF with TNFR1 and/or TNFR2, respectively, led to the conclusion that both TNF receptors might cooperate in TNF-induced inflammatory responses. ${ }^{10,11}$ However, the precise role of TNFR2 in sepsis, where TNFR1 definitely is responsible for the inflammatory reaction as outlined above, has not been clarified. Interestingly, TNFR2-deficient mice were resistant to changes of the immune status induced by sepsis, such as increased susceptibility to subsequent infections $^{12}$ and increased numbers of regulatory T cells. ${ }^{13}$

We used TNFR2-deficient mice to investigate whether TNFR2 might play a decisive role modulating immune responses after cecal ligation and puncture (CLP), a mouse model for induction of poly-microbial peritonitis. Assuming that dendritic cells (DC), which play a central role in linking innate with adaptive immune responses, provide a clue for the function of TNFR2, we compared primary DC from TNFR2deficient and wild type (WT) mice and detected impaired Ag presenting capacity of TNFR2-deficient DC after CLP. This phenotypic characterization of DC should help to mechanistically understand and interpret previous observations made in experimental mouse models using TNFR2-deficient mice.

\section{Material and methods}

\section{Animals}

C57BL/6 mice were purchased from Janvier (Le Genest, France). TNFR2-deficient mice (C57BL/6Tnfrsf $\left.1 b^{\mathrm{tm} 1 \mathrm{Mwm}}\right)^{14}$ were purchased from The Jackson Laboratory (Bar Harbour, ME, USA), and deficiency of TNFR2 expression was verified by PCR. Mice were housed in the animal facility of the University of Regensburg and handled in accordance with institutional guidelines.

\section{Cells}

Peripheral mononuclear cells were isolated from blood by density gradient separation. Blood containing EDTA was placed onto Pancoll (PAN-Biotech, Aidenbach, Germany) and interphase was harvested after centrifugation. Following washing, cells were placed in culture. CD11c-positive cells were isolated by magneticactivated cell sorting (MACS) from splenic single cell suspensions following the manufacturer's protocol (Miltenyi Biotec, Bergisch Gladbach, Germany). For generation of bone marrow-derived DC (BMDC), mice were killed and femura and tibiae dissected, quickly placed in $70 \%$ aqueous ethanol, both ends cut and bone marrow flushed out with PBS. Cells were washed once with medium and re-suspended in RPMI medium containing granulocyte-macrophage colonystimulating factor (GM-CSF) for generation of BMDC as described previously. ${ }^{15}$

\section{CLP}

Mice were anesthetized $(75 \mathrm{mg} / \mathrm{kg}$ Ketanest; Parke, Davis \& Company, Munich, Germany) and $16 \mathrm{mg} / \mathrm{kg}$ Rompun (Bayer AG, Leverkusen, Germany) i.p. The cecum was exteriorized and $30 \%$ of the distal end was ligated and punctured once with a $27-\mathrm{G}$ needle to achieve a sublethal CLP. ${ }^{16}$

\section{Flow cytometry}

Following lysis of red blood cells (RBC) in blood and splenic single cell suspensions, cells were incubated with Abs against CD16/32 to block non-specific Ab binding and stained with fluorochrome-labeled Abs. Intracellular cytokines were detected using the Cytofix/Cytoperm kit (BD Biosciences, Heidelberg, Germany). Fluorochrome- or biotin-labeled Abs detecting mouse CD11c, CD11b, CD4, CD8a, B220, Ly6C, Ly6G, CD86, CD80, IL-12p40, IFN- $\gamma$ and MHC-II, as well as fluorochrome-labeled streptavidin, were purchased from BD Biosciences, eBioscience (Frankfurt, Germany), Miltenyi Biotec (Bergisch Gladbach, Germany) or Invitrogen (Darmstadt, Germany). Cells were measured on a BD LSR-II or a BD FACSCalibur flow cytometer, and analyzed using the FacsDiva or CellQuest softwares (BD Biosciences).

\section{Cytokine production}

Splenic single cell suspensions after lysis of RBC or BMDC were cultured with cell culture medium (RPMI 1640 with 10\% FCS, Pen/Strep, $50 \mu$ M 2-ME) at $37^{\circ} \mathrm{C}$. For detection of IL-12p40-producing cells, cells were cultured for $4 \mathrm{~h}$ in the presence of Monensin (GolgiStop; BD Biosciences), usually stimulated with $5 \mu \mathrm{g} / \mathrm{ml}$ cytosin-guanosin oligonucleotid ODN 1668 (CpG; Metabion, Munich, Germany), 
but without $\mathrm{CpG}$ stimulation in the LPS tolerance experiments. For IL-12 family mRNA detection, cells were stimulated with $\mathrm{CpG}$ for $6 \mathrm{~h}$. ELISA kits were used for quantification of IL-12p70 (Bender MedSystems, Vienna, Austria) in supernatants of 18-h cultures with $\mathrm{CpG}$, and for quantification of IFN- $\gamma$ (R\&D Systems, Wiesbaden, Germany) in serum.

\section{Ag presentation}

CD $11 \mathrm{c}^{+}$cells were isolated from splenocytes by magnetic separation with anti-CD11c beads, and CD4 ${ }^{+} \mathrm{T}$ cells were isolated from splenocytes of OT-II mice with anti-CD4 beads according to the manufacturer's instructions (Miltenyi Biotec). OT-II CD4 ${ }^{+} \mathrm{T}$ cells were labeled with $1 \mu \mathrm{M}$ 5,6-carboxyfluorescein succinimidyl ester (CFSE) and $2 \times 10^{5}$ cells were incubated with $4 \times 10^{4} \mathrm{CD} 11 \mathrm{c}^{+}$cells and varying concentrations of ovalbumin (OVA). CFSE dilution was analyzed after $72 \mathrm{~h}$ of culture by flow cytometry to quantify proliferation.

\section{Quantitative real-time PCR}

RNA was prepared from samples using the Nucleospin RNA II kit according to the manufacturer's instruction (Macherey-Nagel, Düren, Germany) and cDNA was generated by transcription with Moloney murine leukemia virus reverse transcriptase (MMLV), (Promega, Mannheim, Germany). Primers for IL-12p35 mRNA (forward: TGT CAA TCA CGC TAC CTC CTC; reverse: TTT TCT CTG GCC GTC TTC AC), IL12p40 mRNA (forward: TCA GGG ACA TCA TCA AAC CA; reverse: CTT TCT GGT TAC ACC CCT CCT), TNF mRNA (forward: CCC CAA AGG GAT GAG AAG TT; reverse: CAC TTG GTG GTT TGC TAC GA), 18S rRNA (forward: GTA ACC CGT TGA ACC CCA TT; reverse: CCA TCC AAT CGG TAG TAG CG) and $\beta$-actin (forward: TCA CCC ACA CTG TGC CCA TCT ACG A; reverse: GGA TGC CAC AGG ATT CCA TAC CCA) were purchased from Metabion (Martinsried, Germany). Real-time PCR was performed with the iQ SYBR Green Super Mix (BioRad, Munich, Germany) on an iQ5 iCycler (BioRad) with 40 cycles $\left(20 \mathrm{~s}\right.$ at $95^{\circ} \mathrm{C}, 30 \mathrm{~s}$ at $58^{\circ} \mathrm{C}, 30 \mathrm{~s}$ at $\left.72^{\circ} \mathrm{C}\right)$.

\section{Mouse models}

LPS tolerance model. ${ }^{17}$ Mice were challenged $24 \mathrm{~h}$ after pre-treatment with either LPS from Escherichia coli 0127:B8 or human TNF $(0.025 \mathrm{mg} / \mathrm{mouse}$, i.v. $)$ with LPS $(0.1 \mathrm{mg} / \mathrm{mouse}$, i.v.), and spleen was sampled 90 min later.

Propionibacterium acnes sensitizing model. Dead Propionibacterium acnes was injected $(0.02 \mathrm{~g} / \mathrm{kg}$ in $0.2 \mathrm{ml}$ PBS, i.v.) and $7 \mathrm{~d}$ later mice were challenged with LPS from
Salmonella Abortus Equi $(0.01 \mathrm{mg} / \mathrm{kg}$ in $0.2 \mathrm{ml}$ PBS, i.v.). Four $h$ after LPS injection serum IFN- $\gamma$ levels were determined.

Statistics. Unpaired Student's $t$-test or two-way ANOVA with Bonferroni post hoc test were used in experiments with two or more experimental groups. $P<0.05$ was accepted as significantly different. All statistics were performed using GraphPad Prism 5.0 (GraphPad Software, La Jolla, CA, USA). All data shown in figures are representative of 2-3 experiments (except for Figure 2a, which was done once).

\section{Results}

\section{Sepsis reduces lymphocyte population in TNFR2-deficient mice}

First, we analyzed the impact of TNFR2-deficiency on splenic cell populations in naïve mice and after CLP (Table 1). In naïve mice, the only difference between the two mouse lines consisted of lower numbers of splenic CD4 ${ }^{+} \mathrm{T}$ cells in TNFR2-deficient mice. We have previously shown that total cell, B and T cell numbers in the spleen are reduced $2 \mathrm{~d}$ after CLP. ${ }^{18}$ Here, we detected a similar reduction of total splenocytes and splenic lymphocyte numbers after CLP in TNFR2-deficient mice. However, CLP hardly affected the cell numbers of CD11b ${ }^{+}$in TNFR2-deficient mice. $\mathrm{CD} 1 \mathrm{~b}^{+}$cell numbers were significantly higher in septic TNFR2-deficient compared with septic WT mice. This strong difference between WT and TNFR2-deficient mice was induced by CLP in cell populations identified as inflammatory myeloid cells or myeloid-derived suppressor cells [MO-MDSC, identified as $\mathrm{CD}_{11 b^{+}} \mathrm{Ly}_{6 \mathrm{C}^{\mathrm{hi}}} \mathrm{Ly}_{6 \mathrm{G}}$, and polymorphonuclear (PMN)-MDSC, identified as CD11b ${ }^{+}$Ly6C ${ }^{\text {int }}$ Ly $\left.6 \mathrm{G}^{+}\right] .{ }^{19}$ CLP reduced the number of splenic MOMDSC and PMN-MDSC strongly in WT mice, but it did not change the numbers in TNFR2-deficient mice. We also compared activation markers on myeloid $\mathrm{CD}_{11 \mathrm{~b}}{ }^{+}$and $\mathrm{CD} 11 \mathrm{c}^{+}$cells in both mouse lines (Table 2). Two d after sepsis, activation markers on splenic WT $\mathrm{CD} 11 \mathrm{~b}^{+}$cells had a tendency to be increased, as we have observed previously. ${ }^{18}$ This effect was also seen in TNFR2-deficient mice.

\section{Production of IL-I 2 of splenic DC from TNFR2-deficient mice is impaired}

Next, we analyzed the impact of CLP and TNFR2 on DC function by harvesting DC $2 \mathrm{~d}$ after CLP and activating them in vitro. Stimulation of splenic DC with $\mathrm{CpG}$ resulted in lower levels of IL-12p70, as well as in reduced numbers of IL-12p40-producing DC in naïve TNFR2-deficient compared with naïve WT mice (Figure 1a, b). Also, the fraction of IL-12-producing 
Table I. Splenic lymphocyte populations in naive and septic WT and TNFR2-deficient mice.

\begin{tabular}{|c|c|c|c|c|c|c|c|c|}
\hline & \multicolumn{3}{|c|}{ C57BL/6 WT mice $\left(\times 10^{6}\right.$ cells $)$} & \multicolumn{3}{|c|}{ TNFR2-deficient mice $\left(\times 10^{6}\right.$ cells $)$} & \multicolumn{2}{|c|}{ Significance WT/TNFR2-def } \\
\hline & Untreated & CLP & $S$ & Untreated & CLP & $\mathrm{S}$ & Untreated & CLP \\
\hline Total cells & $97.4 \pm 3.9$ & $57.2 \pm 13.3$ & $*$ & $85.6 \pm 10.4$ & $50.8 \pm 12.7$ & $*$ & NS & NS \\
\hline B cells & $48.5 \pm 3.0$ & $18.3 \pm 7.2$ & $*$ & $43.7 \pm 6.7$ & $20.7 \pm 7.5$ & $*$ & NS & NS \\
\hline $\mathrm{CD}^{+}{ }^{\mathrm{T}} \mathrm{T}$ cells & $19.8 \pm 1.4$ & $14.2 \pm 3.7$ & $*$ & $13.8 \pm 1.3$ & $9.8 \pm 1.8$ & $*$ & $*$ & $*$ \\
\hline $\mathrm{CD}^{+} \mathrm{T}_{\text {cells }}$ & $15.0 \pm 1.4$ & $18.5 \pm 2.4$ & $*$ & $12.8 \pm 1.2$ & $11.7 \pm 2.5$ & NS & NS & $*$ \\
\hline $\mathrm{CDIIb}^{+}$cells & $3.86 \pm 0.79$ & $1.68 \pm 0.56$ & $*$ & $4.56 \pm 0.87$ & $4.20 \pm 0.21$ & NS & NS & $*$ \\
\hline $\mathrm{CDIIc}^{+}$cells & $\mathrm{I} .85 \pm 0.42$ & $0.4 I \pm 0.09$ & $*$ & $1.91 \pm 0.20$ & $0.51 \pm 0.08$ & $*$ & NS & NS \\
\hline MO-MDSC & $0.67 \pm 0.19$ & $0.34 \pm 0.13$ & NS & $0.81 \pm 0.20$ & $0.89 \pm 0.06$ & NS & NS & $*$ \\
\hline PMN-MDSC & $0.73 \pm 0.14$ & $0.15 \pm 0.08$ & NS & $0.98 \pm 0.23$ & $1.03 \pm 0.79$ & NS & NS & $*$ \\
\hline
\end{tabular}

Splenocytes were analyzed in untreated mice or $2 \mathrm{~d}$ after CLP. Data are given as mean \pm SD $(n=5-7)$. The columns labeled ' $S$ ' give the statistical significances for untreated versus CLP-treated mice of the respective mouse line; the last column gives the statistical significances between the two mouse lines within the untreated or the CLP-treated mice. Significance was calculated with Bonferroni post hoc test following a two-way ANOVA. NS: no significant effect. $* P<0.05$.

Table 2. Activation markers of myeloid cells in naïve and septic WT and TNFR2-deficient mice.

\begin{tabular}{|c|c|c|c|c|c|c|c|c|}
\hline & \multicolumn{3}{|c|}{ C57BL/6 WT mice } & \multicolumn{3}{|c|}{ TNFR2-deficient mice } & \multicolumn{2}{|c|}{ Significance WT/TNFR2-def } \\
\hline & Untreated & CLP & $\mathrm{S}$ & Untreated & CLP & $S$ & Untreated & CLP \\
\hline \multicolumn{9}{|l|}{$\mathrm{CDIIb} \mathrm{b}^{+}$} \\
\hline MHC-II & $28.9 \pm 4.3 \%$ & $30.2 \pm 5.3 \%$ & NS & $23.8 \pm 3.2 \%$ & $24.3 \pm 5.4 \%$ & NS & NS & NS \\
\hline $\mathrm{CD}_{86}{ }^{+}$ & $13.0 \pm 2.2 \%$ & $17.8 \pm 4.4 \%$ & NS & $10.6 \pm 3.6 \%$ & $16.8 \pm 4.9 \%$ & $*$ & NS & NS \\
\hline $\mathrm{CD} 80^{+}$ & $12.0 \pm 3.5 \%$ & $22.9 \pm 8.2 \%$ & $*$ & $10.2 \pm 4.3 \%$ & $12.8 \pm 4.2 \%$ & NS & NS & $*$ \\
\hline \multicolumn{9}{|l|}{$\mathrm{CDIIc} \mathrm{c}^{+}$} \\
\hline MHC-II ${ }^{+}$ & $49.1 \pm 2.3 \%$ & $40.0 \pm 5.6 \%$ & NS & $43.2 \pm 14.1 \%$ & $45.8 \pm 6.8 \%$ & NS & NS & NS \\
\hline $\mathrm{CD} 6^{+}$ & $22.7 \pm 3.8 \%$ & $26.4 \pm 4.7 \%$ & NS & $22.4 \pm 2.4 \%$ & $29.9 \pm 5.7 \%$ & $*$ & NS & NS \\
\hline $\mathrm{CD} 80^{+}$ & $18.4 \pm 1.5 \%$ & $16.9 \pm 3.4 \%$ & NS & $18.3 \pm 3.7 \%$ & $21.2 \pm 3.7 \%$ & NS & NS & NS \\
\hline
\end{tabular}

Splenocytes were analyzed in untreated mice or $2 \mathrm{~d}$ after CLP. Data are given as mean \pm SD $(n=5-7)$. The columns labeled ' $S$ ' give the statistical significances for untreated versus CLP-treated mice of the respective mouse line; the last column gives the statistical significances between the two mouse lines within the untreated or the CLP-treated mice. Significance was calculated with Bonferroni post hoc test following a two-way ANOVA. NS: no significant effect. $* P<0.05$.

DC was found to be similarly reduced in $\mathrm{CpG}$ stimulated DC from blood of naïve TNFR2-deficient mice (Figure 1c).

Sepsis lowered the percentage of IL-12p40-positive splenic DC and significantly reduced the production of IL-12p70 by DC isolated from spleens of WT mice, as previously described. ${ }^{20,21}$ Even though TNFR2deficient splenic DC started off at a much lower level when derived from naïve mice, an additional sepsisinduced reduction in IL-12p40-positive splenic DC ratio and IL-12p70 production was seen in DC from septic TNFR2-deficient mice (Figure 1a, b). This CLPinduced reduction in IL-12p70 in TNFR2-deficient mice was still significant, while no significant reduction was measured in the percentage of IL-12p40-positive $\mathrm{CD}_{1}{ }^{+}$cells. CpG-induced mRNA expression levels of proteins constituting the IL-12 family (p40, p35, p19, p28 and Ebi 3) were determined. All tested genes, and, in particular, genes coding for p40 and p35, were found to be more strongly expressed in DC from naïve WT than in naïve TNFR2-deficient mice, supporting the IL-12 protein data (Figure 1d and data not shown).

As it was previously shown that CLP leads to epigenetic changes in $\mathrm{DC},{ }^{21}$ we wondered if the reduced IL-12 production by DC from TNFR2-deficient mice is based on similar alterations. To investigate if precursor cells of DC in the bone marrow are already destined to have an IL-12 production deficiency owing to (possibly epigenetic) alterations, BMDC from both mouse lines were generated by culturing bone marrow cells in the presence of GM-CSF. The resulting BMDC from the two mouse lines showed equal IL-12 production after CpG stimulation (data not shown) and not the reduced IL-12 production phenotype seen with splenic DC from TNFR2-deficient mice. The changes in IL-12 production could be based on the lack of TNFR2 on the DC themselves, or on a general change in the microenvironment in TNFR2-deficient mice. In order to clarify 


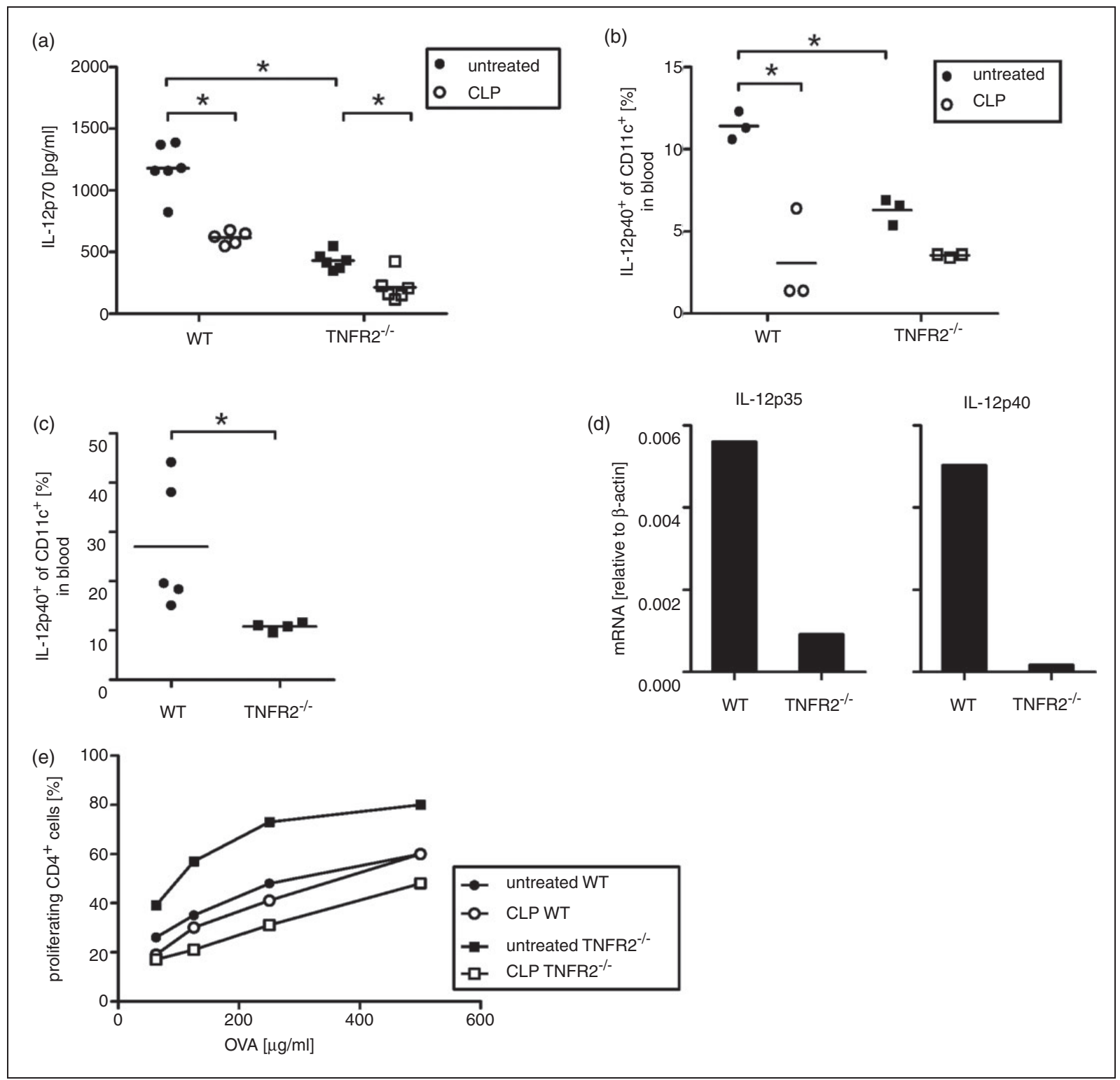

Figure I. WT or TNFR2-deficient mice were either left untreated or treated with CLP (naïve WT: filled circles; CLP-treated WT: open circles; naïve TNFR2-deficient: filled squares; CLP-treated TNFR2-deficient mice: open squares). MACS-purified DC were prepared from splenocytes $2 \mathrm{~d}$ after $\operatorname{CLP}(\mathrm{a}, \mathrm{b}, \mathrm{d})$ or blood cells were prepared from naïve mice (c) and cultured in the presence of CPG. (a) IL-12p70 was determined in supernatant after I8 h of culture. (b, c) IL-12p40+ DC were detected after $4 \mathrm{~h}$ of culture. (a-c) Each symbol represents a mouse; line indicates mean. *Statistically significant difference. (d) mRNA levels were determined after $6 \mathrm{~h}$ of culture. Bars indicate the mean mRNA levels of technical replicates. (e) MACS-purified splenic DC were cultured in presence of CFSElabeled OT-II CD4 ${ }^{+} \mathrm{T}$ cells and varying concentrations of OVA. 3d, proliferation of CD4 ${ }^{+} \mathrm{T}$ cells was analyzed. Symbols indicate the mean of technical replicates.

this, we generated chimeric mice with a TNFR2deficient hematopoietic system in a WT host. Splenic DC of these chimeric mice did not show impaired IL-12 production capacity: splenic DC of WT-in-WT control chimeric mice stimulated ex vivo with $\mathrm{CpG}$ showed $4.6 \pm 0.5 \%$ IL-12p 40 -producing cells, and splenic DC from TNFR2-deficient-in-WT chimeric mice demonstrated $5.1 \pm 1.0 \%$ IL-12p40-producing DC after CpG stimulation. Similarly, BMDC created from these chimeric mice did not show a difference in IL-12production $(261.3+2.3 \mathrm{pg} / \mathrm{ml}$ in WT-in-WT and $225.6+147 \mathrm{pg} / \mathrm{ml}$ IL-12p70 in TNFR2-deficientin-WT). These data indicate that the reduced IL-12 production of splenic DC in TNFR2-deficient mice is an acquired feature and not a genetically pre-determined phenotype. 


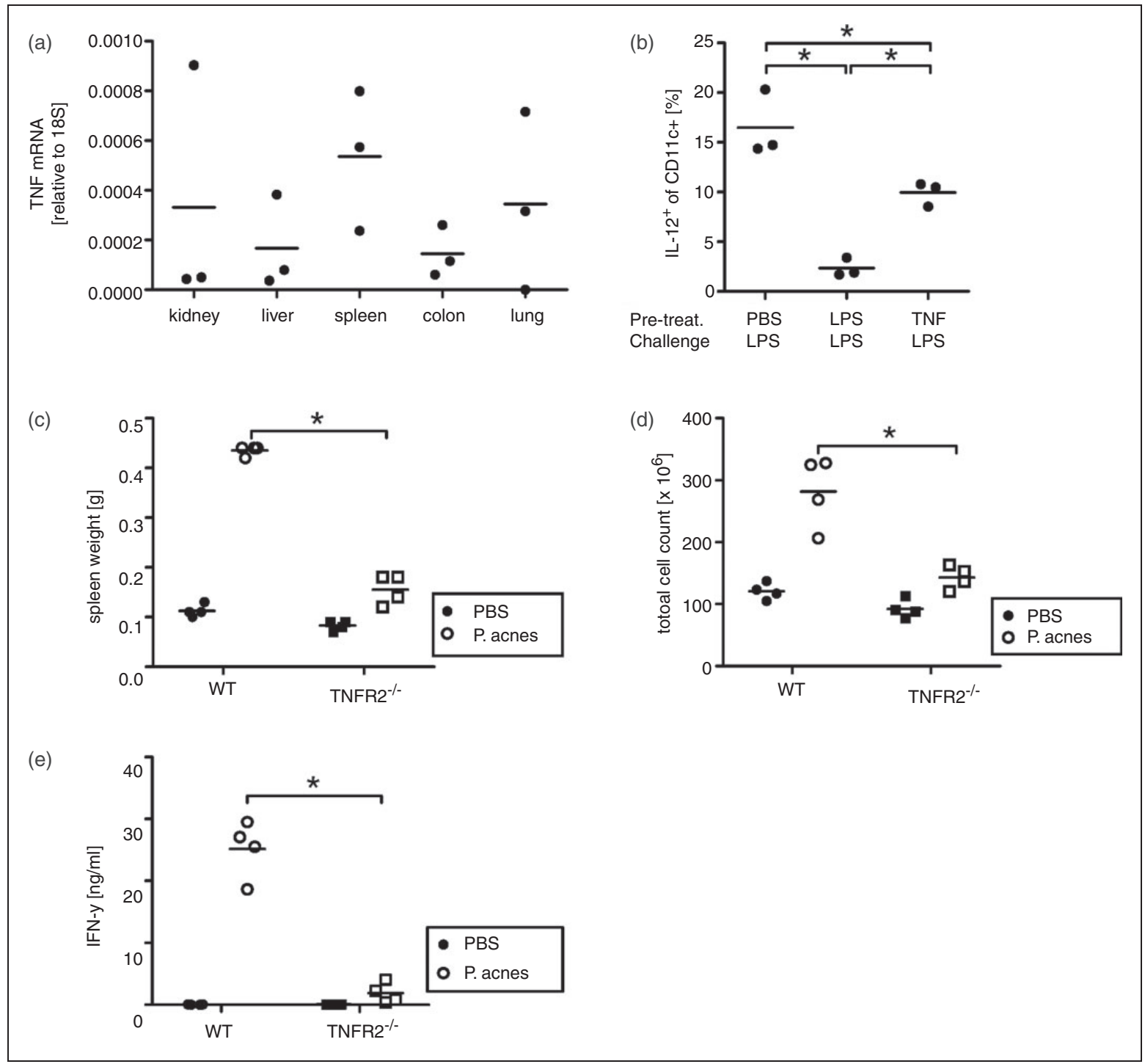

Figure 2. (a) mRNA from indicated tissues of naïve WT mice was analyzed for TNF expression. (b) WT mice were pre-treated (pretreat.) with LPS, TNF or PBS as control and challenged with LPS $24 \mathrm{~h}$ later. Ninety min after challenge, spleens were harvested and IL12-producing cells were determined. (c-e) WT and TNFR2-deficient mice were pre-treated with P. acnes or PBS as a control and challenged with LPS $7 \mathrm{~d}$ later (PBS-treated WT: filled circles; P. acnes-treated WT: open circles; PBS-treated TNFR2-deficient: filled squares; $P$. acnes-treated TNFR2-deficient mice: open squares). Four $\mathrm{h}$ after challenge, spleen masses (a), total cell count in spleens (b) and IFN- $\gamma$ levels in serum were determined. (a-e) Each symbol represents a mouse, line indicates mean. *Statistically significant difference.

\section{Ag presentation is not diminished in TNFR2-deficient DC}

Besides IL-12 production, activation of $\mathrm{T}$ cells is an important function of DC. We have previously shown that sepsis induced by CLP impaired the capacity for Ag (OVA) presentation of splenic DC from WT mice resulting in reduced T cell proliferation. ${ }^{18}$ Surprisingly, Ag-presenting splenic DC from naïve TNFR2-deficient mice showed an enhanced capacity for induction of $\mathrm{T}$ cell proliferation compared to DC from WT mice (Figure 1e). However, CLP markedly reduced the T cell activation capacity of DC from TNFR2-deficient mice, demonstrating a pronounced sepsis-induced suppressive effect by splenic TNFR2-deficient DC. The sepsis-induced shift in proliferation-stimulating activity of DC isolated from TNFR2-deficient mice can not be explained by a clear shift of expression of activation markers on the DC (as shown in Table 2). In contrast to TNFR2-deficient DC, CLP hardly affected the supportive activity of WT DC for CD4 T cell proliferation.

\section{TNF production in healthy mice}

As splenic DC of TNFR2-deficient mice resembled DC from post-septic WT mice in terms of IL-12 production capacity, we searched for the common factor shared 
between septic WT mice and naïve TNFR2-deficient mice. As TNF is strongly increased during sepsis, we wondered whether endogenous bioactive TNF levels might be increased in naïve TNFR2-deficient mice owing to the lack of scavenger-soluble TNFR2. This idea was supported by our recently reported finding of high levels (10-20 ng/ml) of soluble TNFR2 secreted in the urine of naïve WT mice. ${ }^{22}$ In order to determine whether TNF is endogenously produced in WT mice, TNF mRNA expression was measured in different organs of naïve WT mice using TNF-deficient mice as control. The results clearly demonstrated that TNF is expressed in naïve WT mice in a number of tissues (Figure 2a); TNF mRNA was below detection level when isolated from the respective organs from TNFdeficient mice. However, owing to the technical problem of detection of low amounts of protein in urine, we were not able to directly detect endogenous TNF in serum or urine, neither in free form nor bound to TNFR2.

\section{TNF induces habituation in DC}

In order to find out whether exposure to TNF is able to induce a state of tolerance in DC, WT mice were tested in the model of LPS tolerance. ${ }^{23}$ Similar to the changes in DC function observed after CLP, in vivo pretreatment with LPS before LPS challenge reduced the proportion of IL-12-producing splenic DC (Figure 2b). ${ }^{24}$ To validate TNF as an effector molecule in habituation of DC function, we pre-treated WT mice with TNF before LPS challenge. Similar to LPS pretreatment, TNF pre-treatment also reduced the levels of IL-12-producing DC upon LPS challenge (Figure 2b).

\section{DC from TNFR2-deficient mice are refractory to $P$. acnes-induced hyper-sensitization}

Because TNFR2-deficient mice mounted mitigated inflammatory responses compared to WT mice after CLP, we assumed that hyper-sensitization to LPS might also be moderated in these mice. Therefore, we subjected TNFR2-deficient mice to the well-established model for induction of LPS hyper-sensitivity by $P$. acnes injection. ${ }^{25,26}$ As expected, compared with WT mice sensitized with $P$. acnes, TNFR2-deficient mice displayed a significantly smaller splenomegaly upon LPS challenge (Figure 2c, d). IFN- $\gamma$ serum levels were also significantly lower in TNFR2-deficient mice than in WT mice (Figure 2e). Thus, TNFR2deficient mice were not hyper-sensitized by $P$. acnes as strongly as WT mice.

\section{Discussion}

So far, no clear picture of TNFR2 functions has emerged from the use of mice with a genetically ablated
TNFR2 gene. For example, TNFR2-deficient mice have been described to be protected from TNF-induced death and tissue necrosis, ${ }^{14}$ from neurovascular lesions in experimental cerebral malaria, ${ }^{27,28}$ and from experimentally-induced glomerulonephritis. ${ }^{29}$ Cooperative effects of both TNF receptors seemed to aggravate TNF-induced arthritis ${ }^{11}$ and liver toxicity in experimental hepatitis. ${ }^{30}$ In support of these findings, mice over-expressing human TNFR2 displayed increased pathology. ${ }^{31}$ Thus, activation of TNFR2 clearly seemed to contribute to inflammation-induced tissue damage. However, in other models, such as in retinal ischemia $^{32}$ and pulmonary edema, ${ }^{33}$ the lack of TNFR2 exacerbated pathological effects, and selective activation of TNFR2 had a protective effect in oxidative stress-induced neurodegeneration. ${ }^{34}$ Ambiguous functions of TNFR2 were seen in the formation and maintenance of granuloma in mycobacterial infections ${ }^{35}$ and in experimental autoimmune encephalomyelitis where TNFR1 appeared to be required for the initiation of pathology, while TNFR2 was found to exacerbate the disease., ${ }^{56}$ Previous results from our group showed that TNFR2-deficient mice were neither protected from septic death by pre-treatment with LPS $^{37}$ nor did they develop immunoparalysis in sepsis $2 \mathrm{~d}$ after induction by CLP. ${ }^{12}$ These observations pointed to a regulatory role of TNFR2 affecting the immune status.

In this report we present data which show that a mechanism of TNF-induced habituation to inflammatory stimuli seems to operate in TNFR2-deficient mice at the level of myeloid cells. DC from naïve TNFR2deficient mice resembled DC of septic WT mice: septic mice have reduced numbers of functionally impaired DC, ${ }^{18,20,38,39}$ possibly owing to epigenetic regulation. ${ }^{21}$ However, the functionally impaired phenotype of primary DC from naïve TNFR2-deficient mice did not reflect epigenetic modification as TNFR2-deficient BMDC and splenic DC or BMDC from chimeric WT mice with a TNFR2-deficient hematopoietic system were not functionally impaired. Thus, our findings confirm previous reports, i.e. DC isolated from septic mice had an impaired capacity for IL-12 production, ${ }^{40}$ while in vitro-generated DC from such septic mice did not exhibit diminished IL-12 production but developed into regulatory DC with impaired capacity for Th1 priming when injected into mice. ${ }^{41}$ Thus, the impaired function of DC isolated from spleens of TNFR2deficient mice does not seem to be a direct effect of epigenetic alterations in bone marrow DC precursors. Lack of TNFR2 signaling could be a possible cause for the impaired phenotype. In support of this idea was the observation that TNFR2 seemed to provide a survival signal for DC when they were generated in vitro from bone marrow cells of TNFR2-deficient mice. ${ }^{42}$ However, we show that the phenotype of DC from naïve TNFR2-deficient mice resembled DC from septic WT mice. Lack of TNFR2 signaling cannot be 
the reason for the impaired IL-12 response in sepsis as TNFR2 is strongly induced in inflammatory settings. Furthermore, in the well-established model of LPStolerance, naïve WT mice pre-treated with LPS responded in a similar fashion as WT mice $2 \mathrm{~d}$ after CLP with reduced IL-12 production. As both sepsis and LPS pre-treatment cause release of TNF, we investigated the impact of TNF pre-treatment on WT mice. Indeed, WT mice pre-treated with TNF demonstrated a phenotype similar to LPS pre-treated or septic mice with a prominent reduction in IL-12-producing DC.

As we found that TNF induced a refractory state in DC, we tested whether endogenous TNF is present in healthy mice. As direct identification of TNF from urine and serum was below the detection limit, TNF mRNA expression was measured and found in multiple organs. Others have previously shown that endogenous TNF in naïve mice is necessary for the homeostatic development of $\mathrm{T}$ cells without any on-going immune reaction, which clearly demonstrates that biologically active TNF is continuously present in healthy animals. ${ }^{43}$ Together with our previous finding of endogenous soluble TNFR2 secreted with urine from healthy WT mice, ${ }^{22}$ it is plausible that endogenous TNF is constantly sequestered bound to soluble TNFR2. Hence, mice deficient in TNFR2 may continuously or repeatedly become exposed to biologically active, nonsequestered endogenous TNF, thereby training the DC. The training seems to cause a shift in the generation/recruitment of immature myeloid cells (MDSC) as seen in sepsis (Table 1), confounding the maturation of DC in response to inflammatory stimuli. This habituation may also explain the refractoriness of TNFR2-deficient mice to hyper-sensitization by $P$. acnes, in particular, as it has been shown that the IFN- $\gamma$ response to $P$. acnes causing hyper-sensitization critically depends on IL-12. ${ }^{44}$

It is important to note that DC from TNFR2deficient mice were not completely non-functional. DC from naïve TNFR2-deficient mice induced stronger $\mathrm{T}$ cell proliferation than DC obtained from naïve WT mice even though no enhanced expression of activation markers was measured on TNFR2-deficient DC (Table 2). This observation and the fact that CLP induced a clear inversion of the proliferation-stimulating activity of DC isolated from the two mouse lines demonstrate that additional mechanisms such as regulatory cells might be at work for the manifestation of in vivo suppression in sepsis. Interestingly, the appearance of regulatory $\mathrm{T}$ cells in sepsis was impaired in TNFR2deficient mice. ${ }^{13}$ The differential reaction of WT and TNFR2-deficient mice to CLP-induced sepsis strongly reducing the numbers of inflammatory myeloid cells in septic WT mice, whereas no change was induced in TNFR2-derived inflammatory myeloid cell numbers points to a role of these potentially suppressive immature myeloid cells in the habituated phenotype observed with TNFR2-deficient mice. However, whether TNFR2 signaling plays a role in cells participating in this interaction remains to be analyzed.

In conclusion, TNF exposure habituates DC leading to reduced IL-12 responses. This effect is prevented by TNFR2 in naïve mice, but emerges after exposure to high levels of TNF during inflammation or when endogenous TNFR2 is missing. As we have recently shown in the model of immune complex-induced glomerulonephritis, ${ }^{22}$ this training of DC leads to adapted responses following inflammatory reactions. Obviously, previous exposure to endogenous TNF is capable of conferring such a suppression-inducing phenotype on DC in TNFR2-deficient mice. ${ }^{12}$ This calls for a careful interpretation of data obtained with TNFR2-deficient mice as training by endogenous TNF and not (only) lack of TNFR2 signal transduction will impact on the results in such experimental models.

\section{Funding}

This work was supported by a German Research Foundation (DFG) grant Ma760/19-1 (to DNM).

\section{References}

1. Hehlgans $\mathrm{T}$ and Pfeffer $\mathrm{K}$. The intriguing biology of the tumour necrosis factor/tumour necrosis factor receptor superfamily: players, rules and the games. Immunology 2005; 115: 1-20.

2. Grivennikov SI, Kuprash DV, Liu ZG, et al. Intracellular signals and events activated by cytokines of the tumor necrosis factor superfamily: From simple paradigms to complex mechanisms. Int Rev Cytol 2006; 252: 129-161.

3. Marino MW, Dunn A, Grail D, et al. Characterization of tumor necrosis factor-deficient mice. Proc Natl Acad Sci U S A 1997; 94 : 8093-8098.

4. Peschon JJ, Torrance DS, Stocking KL, et al. TNF receptordeficient mice reveal divergent roles for p55 and p75 in several models of inflammation. J Immunol 1998; 160: 943-952.

5. Pfeffer K, Matsuyama T, Kundig TM, et al. Mice deficient for the $55 \mathrm{kd}$ tumor necrosis factor receptor are resistant to endotoxic shock, yet succumb to L. monocytogenes infection. Cell 1993; 73: 457-467.

6. Castanos-Velez E, Maerlan S, Osorio LM, et al. Trypanosoma cruzi infection in tumor necrosis factor receptor p55-deficient mice. Infect Immun 1998; 66: 2960-2968.

7. Tartaglia LA, Pennica D and Goeddel DV. Ligand passing: the 75-kDa tumor necrosis factor (TNF) receptor recruits TNF for signaling by the $55-\mathrm{kDa}$ TNF receptor. J Biol Chem 1993; 268 : 18542-18548.

8. Bigda J, Beletsky I, Brakebusch C, et al. Dual role of the p75 tumor necrosis factor (TNF) receptor in TNF cytotoxicity. $J$ Exp Med 1994; 180: 445-460.

9. Weiss T, Grell M, Hessabi B, et al. Enhancement of TNF receptor p60-mediated cytotoxicity by TNF receptor p80: requirement of the TNF receptor-associated factor-2 binding site. J Immunol 1997; 158: 2398-2404.

10. Chan FK, Shisler J, Bixby JG, et al. A role for tumor necrosis factor receptor-2 and receptor-interacting protein in programmed necrosis and antiviral responses. $J$ Biol Chem 2003; 278: 51613-51621.

11. Alexopoulou L, Kranidioti K, Xanthoulea S, et al. Transmembrane TNF protects mutant mice against intracellular bacterial infections, chronic inflammation and autoimmunity. Eur J Immunol 2006; 36: 2768-2780. 
12. Sterns T, Pollak N, Echtenacher B, et al. Divergence of protection induced by bacterial products and sepsis-induced immune suppression. Infect Immun 2005; 73: 4905-4912.

13. Chen X, Baumel M, Männel DN, et al. Interaction of TNF with TNF receptor type 2 promotes expansion and function of mouse CD4 + CD25 + T regulatory cells. J Immunol 2007; 179: 154-161.

14. Erickson SL, de Sauvage FJ, Kikly K, et al. Decreased sensitivity to tumour-necrosis factor but normal T-cell development in TNF receptor-2-deficient mice. Nature 1994; 372: 560-563.

15. Lutz MB, Kukutsch N, Ogilvie AL, et al. An advanced culture method for generating large quantities of highly pure dendritic cells from mouse bone marrow. J Immunol Methods 1999; 223: 77-92.

16. Pollak N, Sterns T, Echtenacher B, et al. Improved resistance to bacterial superinfection in mice by treatment with macrophage migration inhibitory factor. Infect Immun 2005; 73: 6488-6492.

17. Lehmann V, Freudenberg MA and Galanos C. Lethal toxicity of lipopolysaccharide and tumor necrosis factor in normal and Dgalactosamine-treated mice. J Exp Med 1987; 165: 657-663.

18. Mohr A, Polz J, Martin EM, et al. Sepsis leads to a reduced Agspecific primary Ab response. Eur J Immunol 2012; 42: 341-352.

19. Gabrilovich DI, Ostrand-Rosenberg S and Bronte V. Coordinated regulation of myeloid cells by tumours. Nat Rev Immunol 2012; 12: 253-268.

20. Benjamim CF, Lundy SK, Lukacs NW, et al. Reversal of longterm sepsis-induced immunosuppression by dendritic cells. Blood 2005; 105: 3588-3595.

21. Wen H, Dou Y, Hogaboam CM, et al. Epigenetic regulation of dendritic cell-derived interleukin-12 facilitates immunosuppression after a severe innate immune response. Blood 2008; 111 : 1797-1804.

22. Pfeifer E, Polz J, Griebetal S, et al. Mechanisms of immune complex-mediated experimental glomerulonephritis: possible role of the balance between endogenous TNF and soluble TNF receptor type 2. Eur Cytokine Netw 2012; 23: 15-20.

23. Wysocka M, Robertson S, Riemann H, et al. IL-12 suppression during experimental endotoxin tolerance: dendritic cell loss and macrophage hyporesponsiveness. $J$ Immunol 2001; 166: 7504-7513.

24. Shi M, Deng W, Bi E, et al. TRIM30 alpha negatively regulates TLR-mediated NF-kappa B activation by targeting TAB2 and TAB3 for degradation. Nat Immunol 2008; 9: 369-377.

25. Katschinski T, Galanos C, Coumbos A, et al. Gamma interferon mediates Propionibacterium acnes-induced hypersensitivity to lipopolysaccharide in mice. Infect Immun 1992; 60: 1994-2001.

26. Halpern BN, Prevot AR, Biozzi G, et al. Stimulation of the phagocytic activity of the reticuloendothelial system by Corynebacterium parvum. J Reticuloendothel Soc 1964; 83: 77-96.

27. Lucas R, Juillard P, Decoster E, et al. Crucial role of tumor necrosis factor (TNF) receptor 2 and membrane-bound TNF in experimental cerebral malaria. Eur J Immunol 1997; 27: $1719-1725$.

28. Stoelcker B, Hehlgans T, Weigl K, et al. Requirement for tumor necrosis factor receptor 2 expression on vascular cells to induce experimental cerebral malaria. Infect Immun 2002; 70: 5857-5859.
29. Vielhauer V, Stavrakis G and Mayadas TN. Renal cell-expressed TNF receptor 2, not receptor 1, is essential for the development of glomerulonephritis. J Clin Invest 2005; 115: 1199-1209.

30. Kusters S, Tiegs G, Alexopoulou L, et al. In vivo evidence for a functional role of both tumor necrosis factor (TNF) receptors and transmembrane TNF in experimental hepatitis. Eur $J$ Immunol 1997; 27: 2870-2875.

31. Holtmann MH, Douni E, Schutz M, et al. Tumor necrosis factorreceptor 2 is up-regulated on lamina propria T cells in Crohn's disease and promotes experimental colitis in vivo. Eur J Immunol 2002; 32: 3142-3151.

32. Fontaine V, Mohand-Said S, Hanoteau $\mathrm{N}$, et al. Neurodegenerative and neuroprotective effects of tumor Necrosis factor (TNF) in retinal ischemia: opposite roles of TNF receptor 1 and TNF receptor 2. J Neurosci 2002; 22: RC216.

33. Wilson MR, Goddard ME, O'Dea KP, et al. Differential roles of p55 and p75 tumor necrosis factor receptors on stretch-induced pulmonary edema in mice. Am J Physiol Lung Cell Mol Physiol 2007; 293: L60-L68.

34. Fischer R, Maier O, Siegemund M, et al. A TNF receptor 2 selective agonist rescues human neurons from oxidative stressinduced cell death. PLoS One 2011; 6: e27621.

35. Ehlers S, Kutsch S, Ehlers EM, et al. Lethal granuloma disintegration in mycobacteria-infected TNFRp55-/- mice is dependent on T cells and IL-12. J Immunol 2000; 165: 483-492.

36. Suvannavejh GC, Lee HO, Padilla J, et al. Divergent roles for p55 and p75 tumor necrosis factor receptors in the pathogenesis of MOG(35-55)-induced experimental autoimmune encephalomyelitis. Cell Immunol 2000; 205: 24-33.

37. Echtenacher B and Männel DN. Requirement of TNF and TNF receptor type 2 for LPS-induced protection from lethal septic peritonitis. J Endotoxin Res 2002; 8: 365-369.

38. Efron PA, Martins A, Minnich D, et al. Characterization of the systemic loss of dendritic cells in murine lymph nodes during polymicrobial sepsis. J Immunol 2004; 173: 3035-3043.

39. Flohe SB, Agrawal H, Schmitz D, et al. Dendritic cells during polymicrobial sepsis rapidly mature but fail to initiate a protective Th1-type immune response. J Leukoc Biol 2006; 79: 473-481.

40. Flohe SB, Agrawal H, Flohe S, et al. Diversity of interferon gamma and granulocyte-macrophage colony-stimulating factor in restoring immune dysfunction of dendritic cells and macrophages during polymicrobial sepsis. Mol Med 2008; 14: 247-256.

41. Pastille E, Didovic S, Brauckmann D, et al. Modulation of dendritic cell differentiation in the bone marrow mediates sustained immunosuppression after polymicrobial sepsis. J Immunol 2011; 186: 977-986.

42. Funk JO, Walczak H, Voigtlander C, et al. Cutting edge: resistance to apoptosis and continuous proliferation of dendritic cells deficient for TNF receptor-1. J Immunol 2000; 165: 4792-4796.

43. Chatzidakis I, Fousteri G, Tsoukatou D, et al. An essential role for TNF in modulating thresholds for survival, activation, and tolerance of $\mathrm{CD}^{+} \mathrm{T}$ cells. J Immunol 2007; 178: 6735-6745.

44. Tchaptchet S, Kirberg J, Freudenberg N, et al. Innate, Ag-independent role for $\mathrm{T}$ cells in the activation of the immune system by Propionibacterium acnes. Eur J Immunol 2010; 40: 2506-2516. 